\title{
Inhibition of Calpain Alleviates Apoptosis in Coxsackievirus B3-induced Acute Virus Myocarditis Through Suppressing Endoplasmic Reticulum Stress
}

\author{
Hui Shi, ${ }^{1} \mathrm{PhD}$, Ying Yu, ${ }^{2} \mathrm{PhD}$, Yucheng Wang, ${ }^{1} \mathrm{MSc}$, Xiaoxiao Liu, ${ }^{1} \mathrm{PhD}$, Yong Yu, ${ }^{1}$, Minghui Li, ${ }^{1} \mathrm{PhD}$, \\ Yunzeng Zou, ${ }^{1} \mathrm{PhD}$, Ruizhen Chen, ${ }^{1} \mathrm{PhD}$ and Junbo Ge, ${ }^{1} \mathrm{PhD}$
}

\begin{abstract}
Summary
Virus myocarditis (VMC) is a common cardiovascular disease and a major cause of sudden death in young adults. However, there is still a lack of effective treatments. Our previous studies found that calpain activation was involved in VMC pathogenesis. This study aims to explore the underlying mechanisms further. Neonatal rat cardiomyocytes (NRCMs) and transgenic mice overexpressing calpastatin (Tg-CAST), the endogenous calpain inhibitor, were used to establish VMC model. Hematoxylin and eosin and Masson staining revealed inflammatory cell infiltration and fibrosis. An ELISA array detected myocardial injury. Cardiac function was measured using echocardiography. CVB3 replication was assessed by capsid protein VP1. Apoptosis was measured by TUNEL staining, flow cytometry, and western blot. The endoplasmic reticulum (ER) stress-related proteins were detected by western blot. Our data showed that CVB3 infection resulted in cardiac injury, as evidenced by increased inflammatory responses and fibrosis, which induced myocardial apoptosis. Inhibiting calpain, both by PD150606 and calpastatin overexpression, could attenuate these effects. Furthermore, ER stress was activated during CVB3 infection. However, calpain inhibition could downregulate some ER stress-associated protein levels such as GRP78, pancreatic ER kinase-like ER kinase (PERK), and inositol-requiring enzyme-1 $\alpha$ (IRE-1 $\alpha$ ), and ER stress-related apoptotic factors, during CVB3 infection. In conclusion, calpain inhibition attenuated CVB3-induced myocarditis by suppressing ER stress, thereby inhibiting cardiomyocyte apoptosis.
\end{abstract}

(Int Heart J 2021; 62: 900-909)

Key words: Calcium-dependent proteases, Viral myocarditis, Cell apoptosis, ER stress

V iral myocarditis (VMC) has been considered a primary cause of sudden death in young adults, accounting for about $2 \%-42 \% .^{1)}$ Although most patients could be cured after active symptomatic treatment, about $20 \%$ of patients may gradually develop dilated cardiomyopathy or die due to malignant arrhythmia. ${ }^{2)}$ Moreover, in recent years, the incidence of viral myocarditis has been on the rise, and the fatality rate could be as high as $24 \% .^{3)}$ A variety of viruses could cause VMC. Coxsackievirus B3 (CVB3) is the most common and most extensively studied pathogen clinically. Despite extensive research, there are still challenges to treat VMC in clinics. Therefore, it is necessary to understand the VMC pathogenesis and find new and effective drugs to treat VMC.

Apoptosis is an important pathological process in $\mathrm{VMC}$, and several pro-apoptotic proteins are associated with CVB3 replication. ${ }^{4}$ After affecting cells, the virus damages the myocardium by activating the immune response and aggravating the heart injury through apoptosis. ${ }^{5)}$ In the acute phase of VMC, activating the host's interferon response or viral signaling pathway induces cardiomyocyte apoptosis, which helps release viral progeny to infect more cells. ${ }^{5)}$ In the later stages of infection, inflammatory response and apoptosis co-exist and promote each other. ${ }^{6}$ Inhibiting apoptosis in VMC reportedly has a cardioprotective effect. ${ }^{7-9)}$ Therefore, inhibiting cell apoptosis is also considered a potential treatment for VMC treatment.

The endoplasmic reticulum (ER) is a multifunctional intracellular organelle primarily responsible for protein synthesis, protein folding, protein translocation, calcium homeostasis, and lipid biosynthesis. ${ }^{10)}$ Homeostasis of the ER lumen is critical for folding proteins properly. Perturbations of this homeostasis, caused by any stimuli, could result in misfolding and unfolding proteins, a condition

From the 'Department of Cardiology, Shanghai Institute of Cardiovascular Diseases, Zhongshan Hospital, Shanghai Medical College of Fudan University, Shanghai, China and ${ }^{2}$ Department of General Practice, Zhongshan Hospital, Shanghai Medical College of Fudan University, Shanghai, China.

This work was supported by National Science Foundation of China (No. 81772109, 81521001, 81600294, 31570904, 81671937, 81970315) and Shanghai Sailing Program (No. 20YF1406500).

Address for correspondence: Ruizhen Chen, PhD, Department of Cardiology, Shanghai Institute of Cardiovascular Diseases, Zhongshan Hospital, Shanghai Medical College of Fudan University, 1069 Xietu Road, Shanghai 200032, China. E-mail: chen.ruizhen@zs-hospital.sh.cn

Received for publication December 18, 2020. Revised and accepted January 21, 2021.

Released in advance online on J-STAGE July 6, 2021.

doi: 10.1536/ihj.20-803

All rights reserved by the International Heart Journal Association. 
referred to as ER stress. ${ }^{11)}$ ER stress activates the unfolded protein response (UPR) through signaling pathways to restore cellular homeostasis. ${ }^{12)}$ However, if the UPR fails to restore the cellular homeostasis, cell damages such as cell dysfunction and apoptosis may occur. Many studies have recently found that ER stress is highly associated with cardiovascular diseases such as myocardial ischemia/reperfusion injury, ${ }^{13)}$ diabetic cardiomyopathy, ${ }^{14)}$ and heart failure. ${ }^{15)}$ Meanwhile, many pathogenic microorganisms could cause ER stress, such as some RNA virus-like Infectious Bronchitis Virus, ${ }^{16)}$ West Nile Virus ${ }^{17)}$ and CVB3. ${ }^{18)}$ Moreover, silencing GRP78 (a marker of ER stress) inhibits CVB3 induced apoptosis in cells. ${ }^{18)}$ Suppressing ER stress could alleviate myocardial injury and apoptosis in CVB3induced VMC mice. ${ }^{19-21)}$ These results indicate that targeting ER stress may be a promising therapeutic to decrease cell apoptosis in VMC.

Calpain, a family of calcium-dependent cysteine proteases, is involved in a wide variety of cellular processes, including remodeling of cytoskeletal/membrane attachments, apoptosis, and inflammation. ${ }^{22)}$ Recent studies have revealed that calpain activation could promote ER stress. ${ }^{23)}$ Additionally, our previous studies have demonstrated that calpain activation is involved in pathological processes, such as CVB3 replication, myocardium inflammation, ${ }^{24)}$ NLPP3 inflammasome-medicated pyroptosis, ${ }^{25)}$ implying calpain plays pivotal roles in the VMC. However, whether CVB3 could induce cardiomyocyte apoptosis by activating calpain, thereby promoting the development of $\mathrm{VMC}$, is unclear.

In this study, we used neonatal rat cardiomyocytes and transgenic mice overexpressing calpastatin to investigate calpain's effects on cardiomyocyte apoptosis and ER stress in VMC.

\section{Methods}

Animals: Transgenic mice overexpressing calpastatin ( $\mathrm{Tg}$ CAST) were acquired from the laboratory of Tianqing Peng (Lawson Health Research Institute, Canada) and bred in a standard specific pathogen-free environment in the Department of Laboratory Animal Science of Fudan University. We divided transgenic mice and littermate wild-type (WT) mice (3-4 weeks old) into the following four groups: WT control (Control; $n=8)$, Tg-CAST control (Tg-CAST; $n=8$ ), CVB3-infected (Virus; $n=8$ ), and CVB3-infected Tg-CAST (Tg-CAST+Virus; $n=8$ ) groups. The virus myocarditis animal model was established using Coxsackievirus B3 (CVB3) as described before. ${ }^{24)}$ Briefly, $100,000 \mathrm{TCID}_{50} \times 0.1 \mathrm{~mL} \mathrm{CVB3}$ was injected intraperitoneally into mice. After 7 days, the mice were anesthetized using $1.5 \%$ pentobarbital. Heart tissue was harvested. Blood samples were obtained and centrifuged to isolate serum. The Ethics Committee of Fudan University approved all animal care and treatment procedures.

Histopathology: The hearts were fixed in 4\% paraformaldehyde overnight and embedded in paraffin. The embedded hearts were sliced into $5 \mu \mathrm{m}$-thick sections. The sections were stained with hematoxylin and eosin (H\&E) and Masson according to the manufacturer's protocol. Further- more, immunohistochemical analyses were performed. Sections were incubated with primary antibodies specific for f4/80 (1:800, Servicebio, China), MPO (1:300, Servicebio, China) overnight before secondary antibody (1: 200, Servicebio, China). Stained sections were observed under a microscope (Olympus, Japan).

TUNEL Assay: Hearts were embedded by optimal cutting temperature compound and sliced into $5 \mu \mathrm{m}$-thick sections. TUNEL staining was applied to observe the cell death and were performed by using TUNEL Assay Kit (Roche, Switzerland) according to the manufacturer's instructions.

ELISA: ELISA was used to evaluate the levels of cTnI and $\mathrm{CK}-\mathrm{MB}$ in peripheral blood according to the manufacturer's instructions (Haoben, Shanghai, China).

Echocardiography: Mice were anesthetized with isoflurane, and echocardiography was conducted using an echocardiography system (Vevo Visualsonics 2100, Canada) for 7 days. M-mode parameters were performed to calculate left ventricular ejection fraction (LVEF) and LV fractional shortening (FS).

Cell culture and treatment: Neonatal Sprague-Dawley (SD) rats were sacrificed by decapitation, and the hearts were harvested. Neonatal rat cardiomyocytes (NRCMs) were isolated as previously described. ${ }^{24)}$ The cardiomyocytes were seeded in a six-well plate at a density of $1 \times$ $10^{6}$ cells/well and were cultured in DMEM (HyClone, South Logan, UT, USA) containing $10 \%$ fetal calf serum (Gibco, Grand Island, NY, USA). 5-bromodeoxyuridine (5-BrdU, 0.1 mM) (Sigma-Aldrich, St, Louis, MO, USA) was used to inhibit fibroblast proliferation.

NRCMs were infected with CVB3(Nancy strain) as previously described. PD150606 (MCE, Shanghai, China), a calpain inhibitor, was used at a final concentration of 20 $\mu \mathrm{M}$. Cells were divided into four groups: control group, PD150606 group, Virus group, virus + PD150606 group. After 48 hours, the cells were harvested.

Flow cytometric analysis: An annexin V-FITC apoptosis detection kit (BD, San Jose, CA, USA) was used to detect the NRCMs apoptosis according to the manufacturer's instructions. The analysis was conducted using a fluorescence-activated cell sorting machine with Flow Jo software.

Western blotting analysis: Total proteins were extracted from NRCMs or heart tissue using radioimmunoprecipitation assay buffer (Beyotime, Shanghai, China), and the concentration was determined by a bicinchoninic acid protein assay kit (Beyotime, Shanghai, China). The methods used for western blot were described previously. The membranes were incubated with primary antibodies against $\alpha$-fodrin (1:1000, Enzo Life Sciences, USA.), VP1 (GeneTex, 1:1000, USA), PERK (1:1000, Cell Signaling Technology, USA), p-PERK (1:1000, Invitrogen, USA), IRE-1 $\alpha$ (1:1000, Cell Signaling Technology, USA), pIRE-1 $\alpha$ (1:1000, Cell Signaling Technology, USA.), ATF6 (1:1000, Absin, China), CHOP (1:1000, Cell Signaling Technology, USA), GRP78 (1:1000, Cell Signaling Technology, USA.), Cleaved-Caspase-3 (1:1000, Cell Signaling Technology, USA.), p-Jun N-terminal kinase (p-JNK)(1: 1000, Abclonal, China), caspase-12 (1:1000, Proteintech, UK), Bax (1:1000, Proteintech, UK), Bcl-2 (1:250, Santa 
A

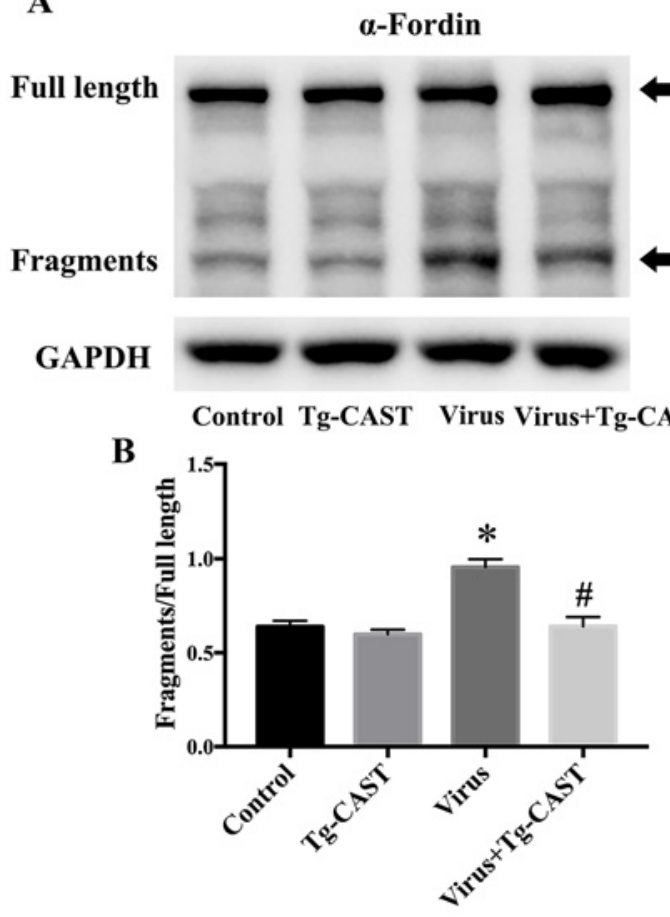

C $250 \mathrm{kDa}$

$150 \mathrm{kDa}$

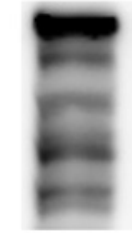
$\alpha$-Fordin

Control PD150606 Virus Virus+PD150606

D

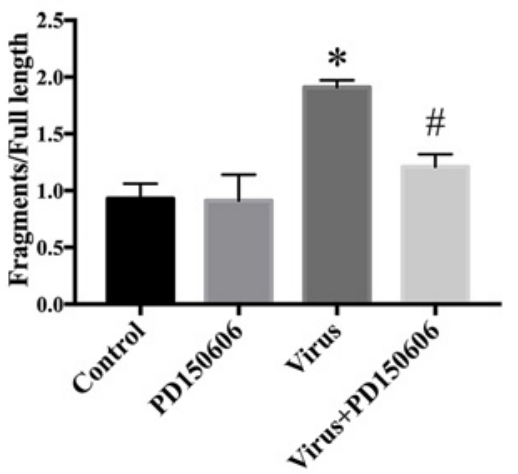

Figure 1. Calpain is activated in CVB3-infected mice. A: The full-length and fragments levels of $\alpha$-fodrin were measured by western blot in heart tissues of each group 7 days after virus injection $(n=8)$. B: The statistical results ratio of protein levels of fragmented $\alpha$-fodrin $(150 \mathrm{kDa})$ and full-length $\alpha$-fodrin $(250 \mathrm{kDa})$ in each group. C: The full-length and fragments levels of $\alpha$-fodrin were measured by western blot in NRCMs (post 48 hours CVB3 infection). D: The statistical results ratio of protein levels of fragmented $\alpha$-fodrin (150 $\mathrm{kDa})$ and full-length $\alpha$-fodrin $(250 \mathrm{kDa})$ in NRCMs. Results were presented as the mean \pm SEM. ${ }^{*} P<0.05$ versus control group, ${ }^{\#} P<$ 0.05 versus virus group. CVB3 indicates Coxsackievirus B3.

Cruz, USA), calpain-1 (1:1000, Cell Signaling Technology, USA), calpain-2 (1:1000, Cell Signaling Technology, USA.), GAPDH (1:2000, Cell Signaling Technology, USA) overnight at $4^{\circ} \mathrm{C}$. Protein bands were incubated with HRP-conjugated secondary antibody (1:5000, Cell Signaling Technology, USA) for 60 minutes at room temperature and visualized by enhanced chemiluminescence (Millipore, Billerica, MA, USA). The quantitative analysis of the blot intensities was performed using Image $\mathrm{J}$ software.

Statistical analysis: Data were presented as mean values \pm SEM. Differences among groups were determined using a one-way analysis of variance. All data were analyzed using GraphPad Prism 7 software. $P<0.05$ was considered statistically significant.

\section{Results}

Calpain is activated during CVB3 infection: $\alpha$-fodrin, the calpain substrates, could be cleaved by activated calpain. ${ }^{26)}$ Therefore, we measured the full-length and fragments levels of $\alpha$-fodrin to indicate calpain activation in CVB3-infected NRCMs and heart tissues. The western blot data showed that the levels of a fragment of $\alpha$-fodrin were higher in the virus group than in the control group, both in vivo and in vitro. However, $\alpha$-fodrin proteolysis was diminished in Tg-CAST mice and PD150606-treated
NRCMs during CVB3 infection (Figure 1A-D). These findings demonstrate that CVB3 infection activates calpain.

Calpain inhibition ameliorates CVB3-induced myocardium injury: The survival curve showed that the virus + Tg-CAST group had a higher survival rate than the virus group (Figure 2A). CVB3 replication plays an important role in VMC pathogenesis. Therefore, we assessed the role of calpain activity in CVB3 replication. As shown in Figure $2 \mathrm{~B}$, inhibition of calpain activity significantly reduced the protein levels of CVB3 capsid protein VP1. H\& E staining inflammatory infiltration was significantly ameliorated in Tg-CAST mice infected with CVB3 (Figure 2 C). During viral infection, cell infiltration consisting of macrophages, neutrophils have been demonstrated, resulting in massive inflammation and aggravated heart injury. ${ }^{27,28)}$ Therefore, we performed immunohistochemical analyses to investigate what kind of inflammatory cells are decreased in the virus $+\mathrm{Tg}$-CAST group. We found that neutrophils and macrophages were lower in virus + Tg-CAST than those in the virus group (Figure 2D, E). Fibrosis is also a key pathological mechanism in VMC. Therefore, we used the Masson statin to observe myocardial fibrosis in transgenic mice. As expected, mice in the virus + Tg-CAST group had fewer areas of fibrotic tissue than mice in the virus group (Figure $2 \mathrm{~F}$ ).

Next, we examined the levels of CK-MB and cTnI in 
A

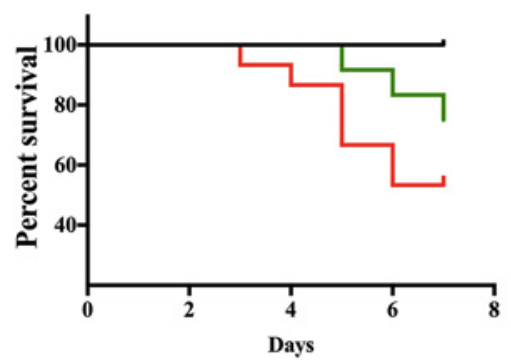

C

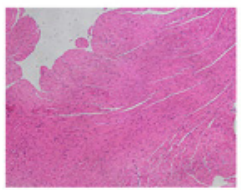

Control

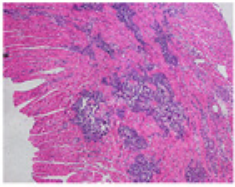

Virus

F

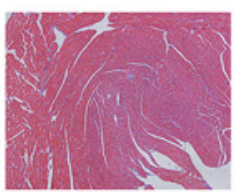

Control

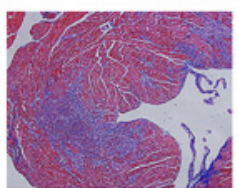

Virus

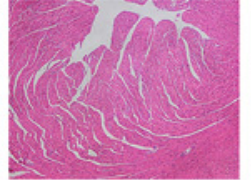

Tg-CAST

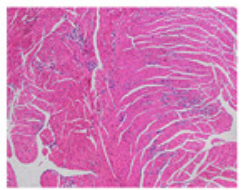

Virus+Tg-CAST

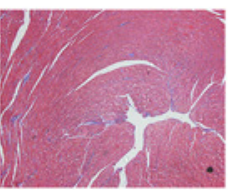

Tg-CAST

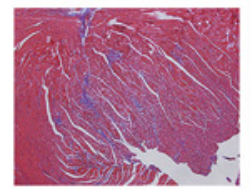

Virus+Tg-CAST
D

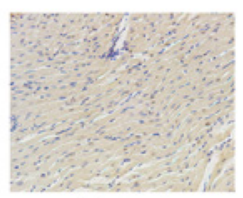

Control

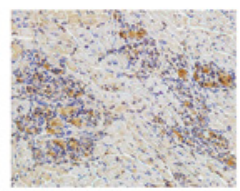

Virus

G

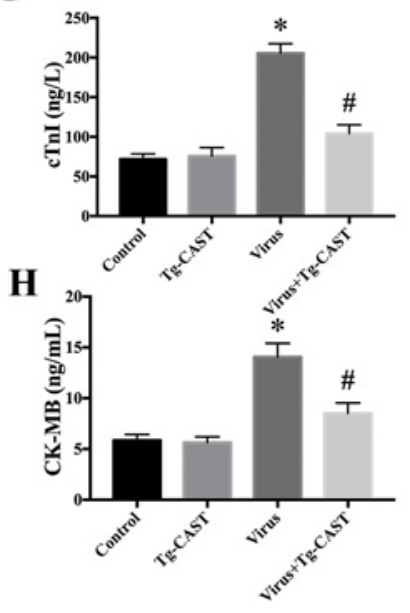

Tg-CAST
B

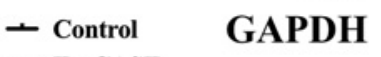

- Tg-CAST

- Virus+Tg-CAST
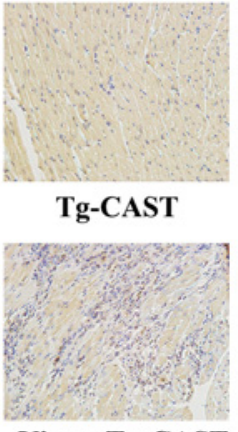

Virus+Tg-CAST
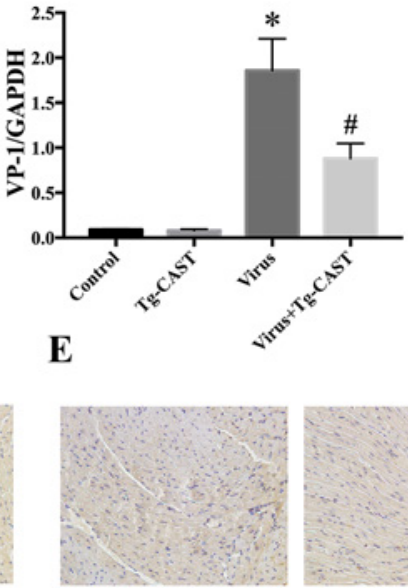

Control

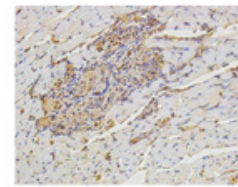

Virus

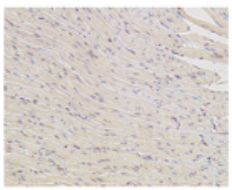

Tg-CAST

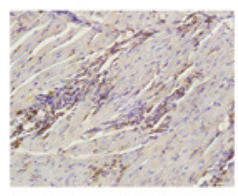

Virus+Tg-CAST

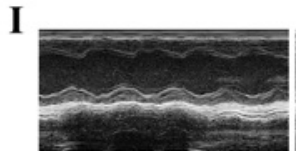

Control

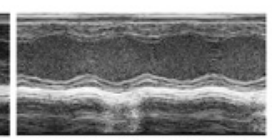

Tg-CAST
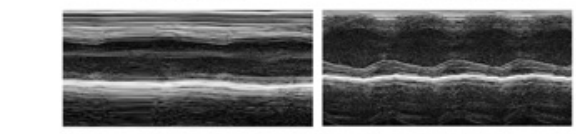

Virus

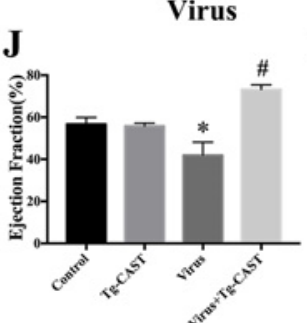

Virus+Tg-CAST

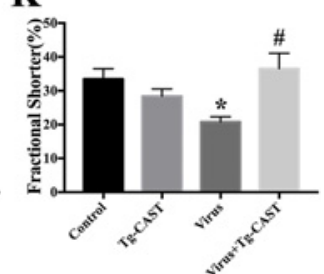

Figure 2. Calpain inhibition ameliorates CVB3-induced myocardium injury. A: The survival rate was measured every day for 7 days. B: The protein expression of capsid protein VP1 was measured by western blot to indicate the CVB3 replication in heart tissues of each group. C: H\&E staining was used to reveal cardiac inflammation in VMC mice (magnification: $\times 100$ ). D: The representative pictures of immunohistochemical analysis of macrophage marker F4/80. E: The representative pictures of immunohistochemical analysis of neutrophil marker MPO. F: Masson staining was used to observe the fibrosis in VMC mice (magnification: $\times 100)$. G, H: The markers of myocardium injury cTnI $(\mathbf{G})$ and CK-MB (H) were detected on day 7 after CVB3 infection in each group. I-K: The representative images of echocardiographic parameters of each group; the statistical results of $\operatorname{LVEF}(\mathbf{J})$ and FS $(\mathbf{K})$ of each group. Results were presented as the mean \pm SEM. $* P<0.05$ versus control group, ${ }^{\#} P<0.05$ versus virus group. CVB3 indicates coxsackievirus B3; LVEF, left ventricular ejection fraction; and FS, fractional shortening.

peripheral blood, two myocardial injury markers. These two markers were elevated in the virus group, while $\mathrm{CK}$ $\mathrm{MB}$ and cTnI levels were reduced in the virus $+\mathrm{Tg}-$ CAST group (Figure 2G, H).
We also evaluated the effect of calpastatin overexpression on cardiac function in VMC mice. Compared with the WT control group, mice in the virus group had lower LVEF and LVFS. In contrast, inhibiting calpain ac- 
tivity by calpastatin overexpression could improve systolic function significantly in mice infected with CVB3 manifested as higher LVEF and LVFS (Figure 2I-K). We also measured the levels of IVSd, LVEDd in each group, but there was no statistical significance between the control and the virus groups (data not shown).

These findings confirm that inhibition of calpain activity ameliorates CVB3-induced myocardium injury and has a protective role in $\mathrm{VMC}$ mice.

Calpain inhibition reduced apoptosis in CVB3-infected mice: Apoptosis is an important index of $\mathrm{VMC}^{29)}$ The results of TUNEL staining showed that there were more apoptotic cells in the heart tissues of mice in the virus group comparing with mice in the WT control group. However, calpastatin overexpression could significantly reduce cell apoptosis in CVB3-induced myocarditis mice (Figure 3A, B). We also evaluated the apoptosis-related protein in each group. The western blot results indicated that calpastatin overexpression could decrease the protein levels of Bax and cleaved-caspase- 3 markedly while increasing the Bcl-2 level in VMC mice (Figure 3C-F). These data suggest that calpain inhibition could inhibit cell apoptosis in CVB3-infected VMC mice.

Calpain inhibition suppressed ER stress in CVB3infected mice: GRP78 is an important component in ER, widely used as a marker of ER stress. ${ }^{30)}$ Thus, we tested the expression of GRP78 in heart tissues of VMC mice. The result exposed that CVB3 infection increased the GRP78 in the virus group, suggesting that CVB3 infection triggered ER stress in VMC mice. However, calpain inhibition by calpastatin overexpression led to lower GRP78 expression in CVB3 infected mice than in the virus group. Additionally, UPR is mediated by three signal transducers: PERK, inositol-requiring enzyme- $1 \alpha$ (IRE- $1 \alpha$ ), and activating transcription factor 6 (ATF6). The western blot showed that CVB3 infection increased the protein levels of p-PERK, p-IRE-1 $\alpha$, and ATF6, while calpastatin overexpression diminished p-PERK and p-IRE- $1 \alpha$ in CVB3infected mice (Figure 4A-F).

CHOP plays an important role in ER stress-induced apoptosis. ${ }^{31)}$ The western blot data showed that CVB3 infection significantly increased the protein level of CHOP in the virus group. However, calpastatin overexpression decreased the expression levels of CHOP (Figure 4A, G). Moreover, caspase-12 and JNK are two other important players in ER stress-induced apoptosis. ${ }^{32}$ Our present study also found that calpastatin overexpress could reduce the expression levels of these proteins (Figure 4A, G, H).

PD150606 ameliorated CVB3-induced ER stressrelated NRCMs apoptosis: First, we performed flow cytometry to observe the antiapoptotic effects of PD150606 (calpain inhibitor) in CVB3-infected NRCMs. The results showed that PD150606 reduced apoptosis in NRCMs during CVB3 infection (Figure 5A, B). Moreover, PD150606 treatment inhibited CVB3 replication as evidenced by decreasing the level of VP-1 in CVB3 infected NRCMs (Figure 5C, D). Next, we examined several proteins associated with ER stress. The protein levels of p-PERK, pIRE- $1 \alpha$, and ATF6 were elevated in the virus group, indicating that ER stress was activated in CVB3-infected NRCMs. The protein levels of p-PERK, p-IRE- $1 \alpha$ were decreased in the virus + PD150606 group. However, similar to in vivo experiment, PD150606 treatment could not reduce the protein expression of ATF6 in CVB3 infected NRCMs (Figure 5C, E-H). Furthermore, the protein expressions of CHOP, cleaved-Caspase-12, and p-JNK were lower in the virus + PD150606 group than that in the virus group (Figure 5C, I-K).

The calpain family has 15 identified isoforms. Among them, calpain-1 and calpain-2 are two isoforms of calpain, majorly expressed in cardiomyocytes. ${ }^{33)}$ Therefore, we measured these two subtypes of calpain in NRCMs. We found that calpain-1 and calpain- 2 were elevated during CVB3 infection, while the protein expressions of calpain-a and calpain-2 were lower in virus + PD150606 group Virus group (Figure 5L-N).

These data indicate that calpain inhibition could reduce ER stress-induced apoptosis during CVB3 infection.

\section{Discussion}

In the present study, we found that calpain was activated in VMC mice, accompanied by increased cell apoptosis and activation of ER stress. Furthermore, inhibition of calpain activation could alleviate myocardium injury, restore cardiac function, prevent apoptosis, decrease the levels of GRP78, p-PERK, p-IRE-1 $\alpha$, CHOP, cleavedCaspase-12, and p-JNK. Based on the results, we concluded that inhibition of calpain attenuated apoptosis in CVB3-infected mice through suppressing ER stress.

Lines of evidence have suggested that calpain is a potential target in many diseases. For example, sustained calpain activation is involved in heart failure development. ${ }^{34)}$ Calpastatin overexpression protects against traumatic spinal cord injury, ${ }^{35)}$ and calpain knock-out attenuates acute kidney injury. ${ }^{30)}$ CVB3 infection could induce calcium overload. ${ }^{36)}$ Excessive calcium induces calpain activation. ${ }^{37}$ Therefore, we hypothesized that CVB3 infection could activate calpain. In our previous studies, we have demonstrated that calpain was activated during CVB3 infection. Furthermore, inhibition calpain activation by calpain synthetic inhibitor ALLN or overexpression calpastatin, an endogenous calpain inhibitor, could inhibit CVB3 replication, attenuate inflammation and suppress NRLP3 inflammasome-medicated pyroptosis. ${ }^{25)}$ Consist with the above studies, we found calpastatin overexpression reduced inflammatory response and fibrosis, alleviated myocardium injury, and restored cardiac function in VMC mice, implying calpain inhibition has a protective role in CVB3-infected mice.

Apoptosis plays a major role in CVB3-induced cell damage. Studies have revealed that cell apoptosis occurs in the heart tissue at the early stage of CVB3 infection. In the latter stage of infection, immunological injury causes the damage of cardiomyocytes through cell apoptosis.5) Cardiomyocytes apoptosis also results in adverse myocardial remodeling and fetal heart failure." Furthermore, apoptotic DNA fragments were found in the hearts of patients who suffered from sudden death due to VMC. ${ }^{6}$ ) These researches suggest that apoptosis is involved in the whole course of VMC. Therefore, anti-apoptosis is a promising option for treating VMC. However, the relation- 
A

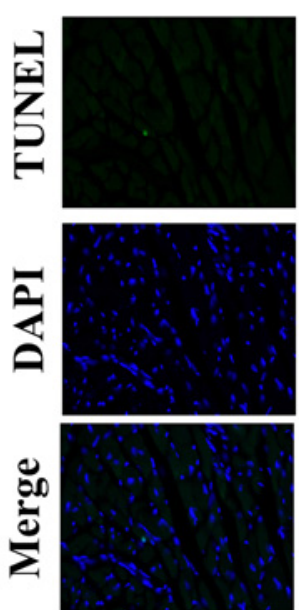

Control
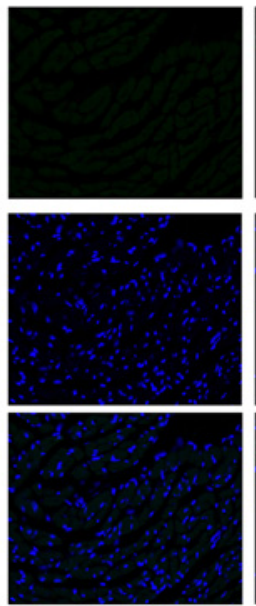

Tg-CAST
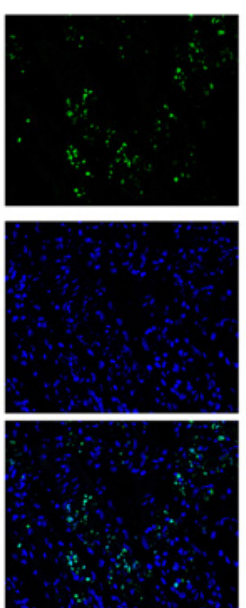

Virus Virus+Tg-CAST

B

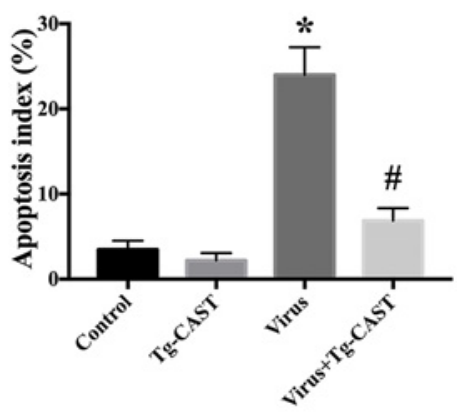

D

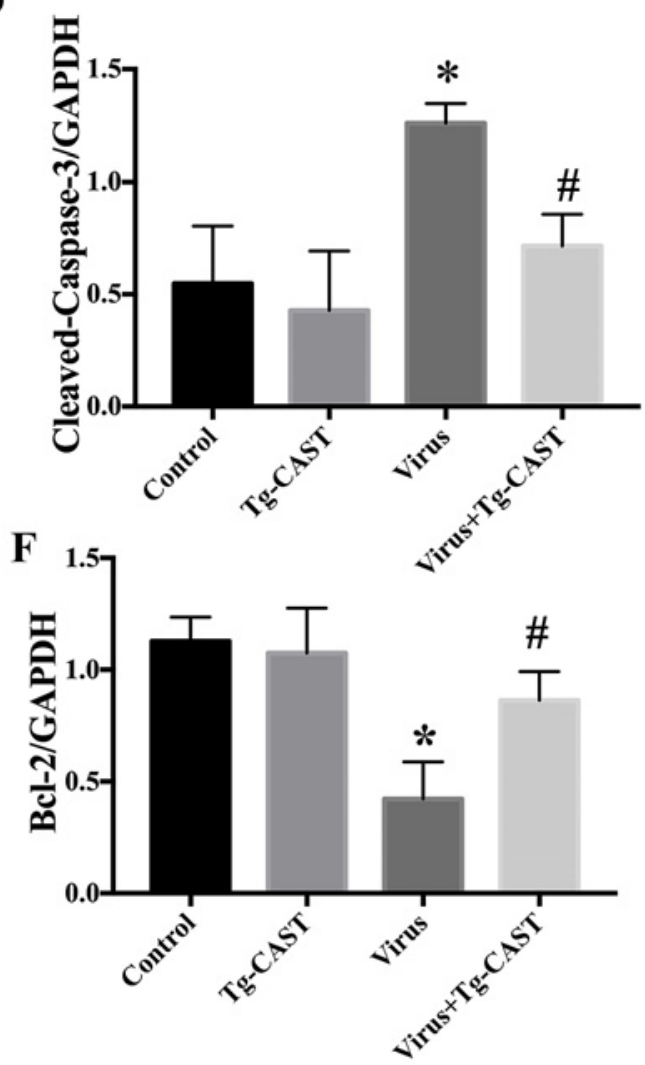

Figure 3. Calpain inhibition reduced apoptosis in CVB3-infected mice. A: Representative images of TUNEL staining in heart tissues of each group. TUNLE-positive apoptotic nuclei were stained with green fluorescence. The nuclei were stained with DAPI with blue fluorescence (magnification: $\times 100$ ). B: Apoptotic index measured from 100 nucleus in a representative area. C: The apoptosis-related proteins were measured by western blot. D-F: The statistical results of protein levels of Cleaved-Caspase-3 (D), Bax (E) and Bcl-2 (F) of each group. Results were presented as the mean \pm SEM. ${ }^{*} P<0.05$ versus control group, ${ }^{\#} P<0.05$ versus virus group. CVB3 indicates coxsackievirus B3.

ship between calpain activation and cell apoptosis is unclear.

Calpain is involved in many pathological processes, including apoptosis. ${ }^{22)}$ Excessive activation of calpain will proteolyze many proteins related to cell apoptosis and promote cell apoptosis. ${ }^{38)}$ Our present study has also found 

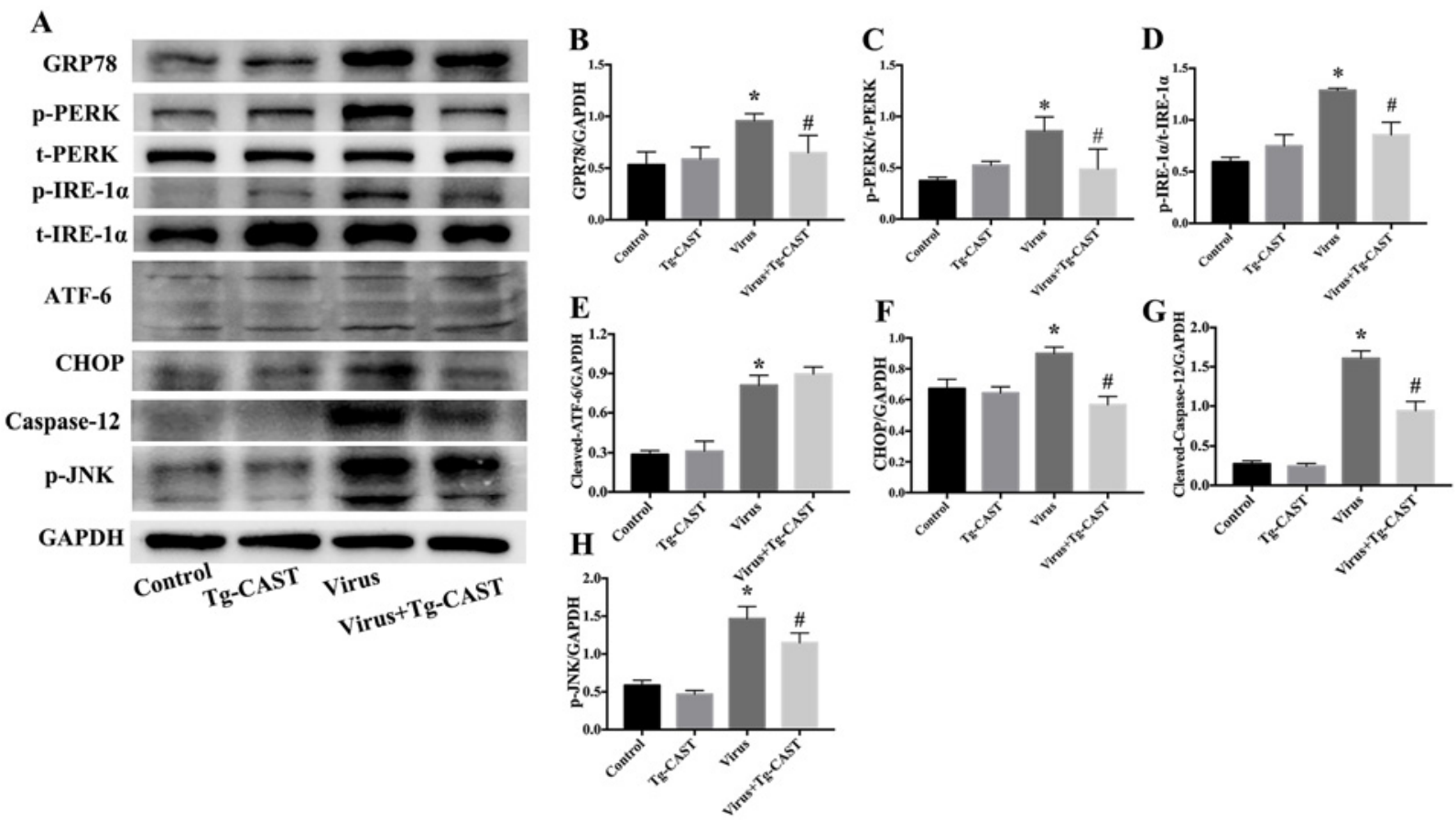

Figure 4. Calpain inhibition suppressed ER stress in CVB3-infected mice. A: The ER stress-related proteins were measured by western blot. B-H: The statistical results of protein levels of GRP78 (B), p-PERK (C), p-IRE-1 $\alpha$ (D), ATF6 (E), CHOP (F), cleaved-Caspase-12 (G), and p-JNK (H) of each group. Results were presented as the mean \pm SEM. ${ }^{*} P<0.05$ versus control group, ${ }^{\#} P<0.05$ versus virus group. CVB3 indicates coxsackievirus B3; p-PERK indicates pancreatic ER kinase-like ER kinase; p-IRE-1 $\alpha$, p-inositol-requiring enzyme-1 $\alpha$; ATF6, activating transcription factor 6; and CHOP, C/EBP homologous protein.

that calpain activation could promote apoptosis in the VMC model. Apoptosis triggered by ER stress is widely studied. Prolonged ER stress could directly induce cell apoptosis by activating JNK, CHOP, and the caspase-12 pathway. ${ }^{32}$ CHOP could regulate numerous apoptotic genes. For example, CHOP overexpression could downregulate $\mathrm{Bcl}-2$ but upregulate caspase-3, Bax, GADD34, and some other pro-apoptotic protein. ${ }^{39)}$ Caspase-12 is an ER membrane-associated cysteine protease and is activated in response of ER stress during CVB3 infection. ${ }^{18)}$ JNK could be activation by IRE1-TRAF2-ASK1 and then causing Bax-dependent cell death. ${ }^{32)}$ Moreover, ER stressinduced apoptosis plays an important role in CVB3induced VMC. ${ }^{18)}$ Cai, et al. revealed that TUDCA (pharmacological ER stress inhibitor), or genetic ablation of CHOP, prevented cardiomyocytes apoptosis in VMC mice by suppressing $\mathrm{Bcl}-2 / \mathrm{Bax}$ ratio and caspase-3 activation. ${ }^{40)}$ JNK could also be phosphorylated by CVB3 infection. ${ }^{41)}$ Consisted with other studies, our present study also found that these factors were upregulated during CVB3 infection. However, the mechanism by which CVB3 induces apoptosis through ER stress is not fully understood.

Recent studies have revealed the relationship between calpain and ER stress. During cellular stress, $\mathrm{Ca}^{2+}$ released from ER could be taken up by mitochondria, producing ROS. ROS could further target ER-based calcium channels by the further release of $\mathrm{Ca}^{2+}$. The sustained high concentration of intracellular $\mathrm{Ca}^{2+}$ activates calpain, which medicated the subsequent ER-mitochondrial crosstalk. Therefore, activation of ER stress is correlated with cal- pains. ${ }^{42)}$ Calpain-1 activation reportedly promotes ER stress and subsequently induces cardiomyocytes apoptosis following hypoxia/reoxygenation. ${ }^{43)}$ Zou, et al. reported that $\mathrm{m}$-calpain could cleave and activate caspase-12, promoting ER stress-induced apoptosis in cardiomyocytes. ${ }^{23)}$ Yan, et al. found that ER stress inhibitor TUDCA treatment did not influence calpain activity in uric acid-treated cardiomyocytes, indicating that calpain is an upstream regulator of ER stress in cardiomyocytes. ${ }^{44)}$ Consist with these studies, our present study has shown that overexpressing calpastatin and PD150606 treatment could reduce the expression of ER stress marker GRP78. Moreover, calpastatin overexpression attenuates myocardial injury in myocardial infarction by suppressing ER stress. ${ }^{45)}$ In the present study, we found inhibition calpain activity decreased CHOP levels, cleaved-Caspase-12, and p-JNK in VMC mice heart tissue. We also measured the protein of the upstream regulatory pathway of these proteins. We found that PERK and IRE- $1 \alpha$, but not ATF- 6 , decreased with calpain inhibition. The results suggested that inhibition of calpain suppressed ER stress-induced apoptosis may by PERK and IRE- $1 \alpha$ but not through ATF6.

In summary, our study demonstrates that calpain inhibition attenuated CVB3-induced myocarditis by suppressing ER stress, thereby alleviating myocardium injury and inhibiting cardiomyocyte apoptosis. This finding provides a promising therapeutic target for VMC. 
A

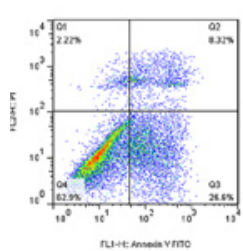

Control

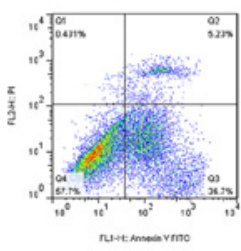

PD150606

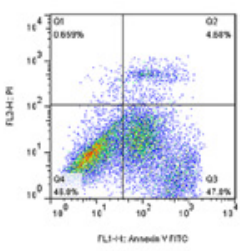

Virus

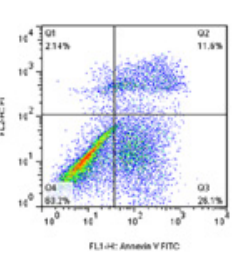

Virus+PD150606
B

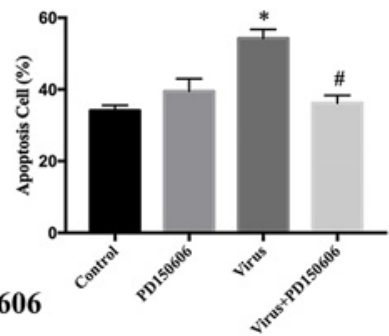

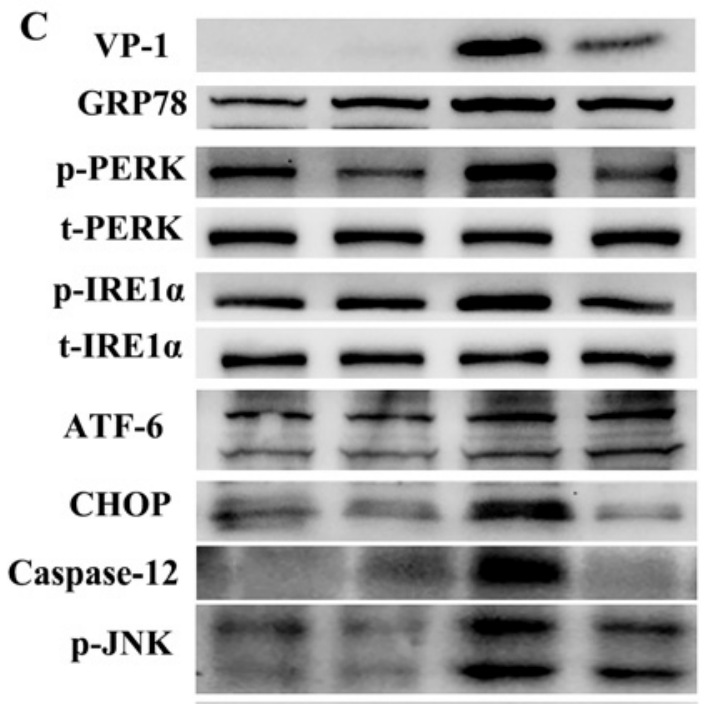

GAPDH

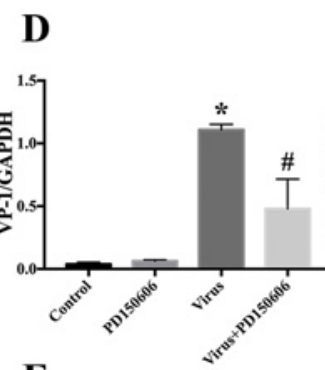

$\mathbf{E}$
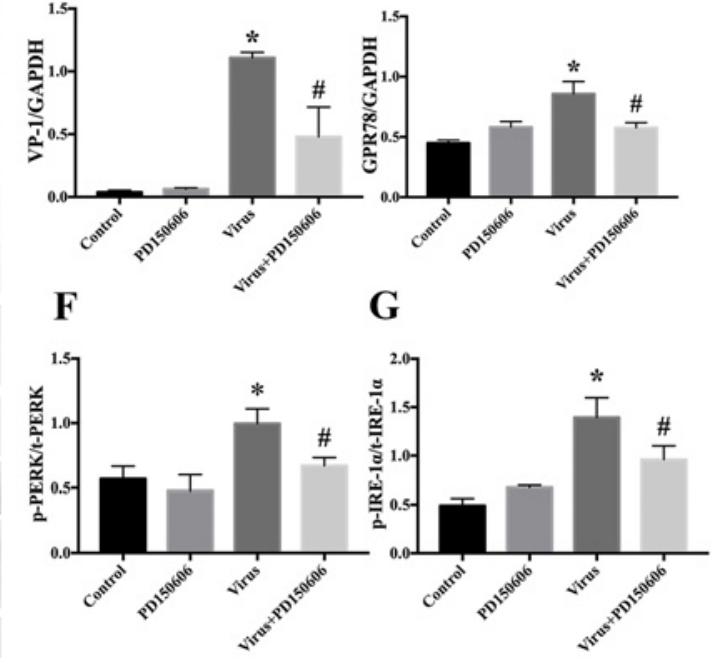

G

Control PD150606 Virus Virus+PD150606

H

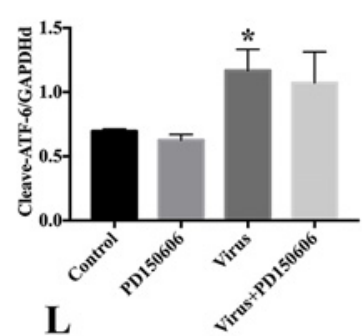

I

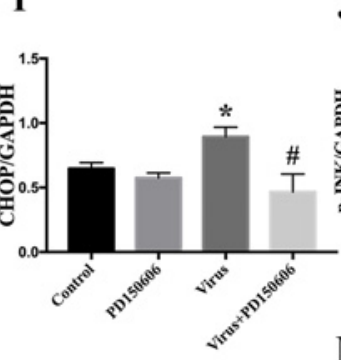

J

M

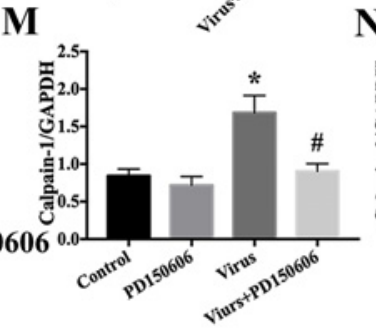

K
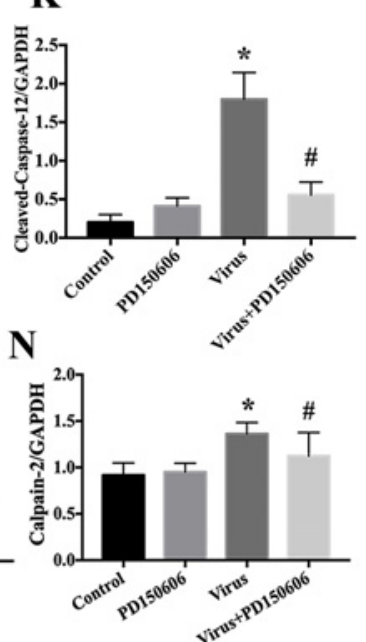

Figure 5. PD150606 ameliorated CVB3-induced ER stress-related NRCMs apoptosis. A, B: the apoptosis was determined by annexin V-FITC/PI staining assay. C: VP-1 and ER stress-related proteins were measured by western blot. D-K: The statistical results of protein levels of VP-1 (D), GRP78 (E), p-PERK (F), p-IRE-1 $\alpha(\mathbf{G})$, ATF6 (H), CHOP $(\mathbf{I})$, p-PNK $(\mathbf{J})$ and cleavedCaspase-12 (K) of each group. L: Calpain-1 and calpain-2 were measured by western blot. M, N: The statistical results of protein levels of calpain-1 (M) and calpain-2 $(\mathbf{N})$ of each group. Results were presented as the mean \pm SEM. $* P<0.05$ versus control group, ${ }^{\#} P<0.05$ versus virus group. CVB3 indicates coxsackievirus B3; p-PERK indicates pancreatic ER kinase-like ER kinase; p-IRE-1 $\alpha$, p-inositol-requiring enzyme-1 $\alpha$; ATF6, activating transcription factor 6; and CHOP, C/EBP homologous protein. 


\section{Disclosure}

Conflicts of interest: None.

\section{References}

1. Caforio ALP, Pankuweit S, Arbustini E, et al. Current state of knowledge on aetiology, diagnosis, management, and therapy of myocarditis: a position statement of the European Society of Cardiology Working Group on myocardial and pericardial diseases. Eur Heart J 2013; 34: 2636-48.

2. Kindermann I, Barth C, Mahfoud F, et al. Update on myocarditis. J Am Coll Cardiol 2012; 59: 779-92.

3. Frasure SE, Siadecki SD, Saul T, Lewiss RE. Viral myocarditis leading to acute heart failure in a young adult. J Emerg Med 2014; 46: e75-7.

4. Colston JT, Chandrasekar B, Freeman GL. Expression of apoptosis-related proteins in experimental Coxsackievirus myocarditis. Cardiovasc Res 1998; 38: 158-68.

5. Corsten MF, Schroen B, Heymans S. Inflammation in viral myocarditis: friend or foe? Trends Mol Med 2012; 18: 426-37.

6. Saraste A, Arola A, Vuorinen T, et al. Cardiomyocyte apoptosis in experimental Coxsackievirus B3 myocarditis. Cardiovasc Pathol 2003; 12: 255-62.

7. Li P, Yan Y, Shi Y, et al. Nicotinic agonist inhibits cardiomyocyte apoptosis in CVB3-induced myocarditis via $\alpha 3 \beta 4-\mathrm{nAChR} /$ PI3K/Akt-dependent survivin upregulation. Oxid Med Cell Longev 2019; 2019: 9496419.

8. Sang Y, Gu X, Pan L, et al. Melatonin ameliorates Coxsackievirus B3-induced myocarditis by regulating apoptosis and autophagy. Front Pharmacol 2018; 9: 1384.

9. Wu R, Wu T, Li P, et al. The protection effects of survivin in the cell model of CVB3-induced viral myocarditis. Heart Vessels 2020; 35: 1171-9.

10. Wang S, Binder P, Fang Q, et al. Endoplasmic reticulum stress in the heart: insights into mechanisms and drug targets. $\mathrm{Br} \mathrm{J}$ Pharmacol 2018; 175: 1293-304.

11. Zhang C, Syed TW, Liu R, Yu J. Role of endoplasmic reticulum stress, autophagy, and inflammation in cardiovascular disease. Front Cardiovasc Med 2017; 4: 29.

12. Liu MQ, Chen Z, Chen LX. Endoplasmic reticulum stress: a novel mechanism and therapeutic target for cardiovascular diseases. Acta Pharmacol Sin 2016; 37: 425-43.

13. Zeng J, Jin Q, Ruan Y, et al. Inhibition of TGFbeta-activated protein kinase 1 ameliorates myocardial ischaemia/reperfusion injury via endoplasmic reticulum stress suppression. J Cell Mol Med 2020; 24: 6846-59.

14. Sun S, Yang S, An N, et al. Astragalus polysaccharides inhibits cardiomyocyte apoptosis during diabetic cardiomyopathy via the endoplasmic reticulum stress pathway. J Ethnopharmacol 2019; 238: 111857

15. Wei SG, Yu Y, Weiss RM, Felder RB. Endoplasmic reticulum stress increases brain MAPK signaling, inflammation and reninangiotensin system activity and sympathetic nerve activity in heart failure. Am J Physiol Heart Circ Physiol 2016; 311: H871-80.

16. Fung TS, Liao Y, Liu DX. The endoplasmic reticulum stress sensor IRE1alpha protects cells from apoptosis induced by the coronavirus infectious bronchitis virus. J Virol 2014; 88: 1275264.

17. Ambrose RL, Mackenzie JM. West Nile virus differentially modulates the unfolded protein response to facilitate replication and immune evasion. J Virol 2011; 85: 2723-32.

18. Zhang HM, Ye X, Su Y, et al. Coxsackievirus B3 infection activates the unfolded protein response and induces apoptosis through downregulation of p58IPK and activation of CHOP and SREBP1. J Virol 2010; 84: 8446-59.

19. Han JY, Jeong HI, Park CW, et al. Cholic acid attenuates ER stress-induced cell death in Coxsackievirus-B3 infection. J Mi- crobiol Biotechnol 2018; 28: 109-14.

20. Ouyang H, Zhong J, Lu J, Zhong Y, Hu Y, Tan Y. Inhibitory effect of melatonin on Mst1 ameliorates myocarditis through attenuating ER stress and mitochondrial dysfunction. J Mol Histol 2019; 50: 405-15.

21. Wang Y, Sun Y, Fu Y, et al. Calumenin relieves cardiac injury by inhibiting ERS-initiated apoptosis during viral myocarditis. Int $\mathbf{J}$ Clin Exp Pathol 2017; 10: 7277-84.

22. Dókus LE, Yousef M, Bánóczi Z. Modulators of calpain activity: inhibitors and activators as potential drugs. Expert Opin Drug Discov 2020; 15: 471-86.

23. Zuo S, Kong D, Wang C, et al. CRTH2 promotes endoplasmic reticulum stress-induced cardiomyocyte apoptosis through $\mathrm{m}$ calpain. EMBO Mol Med 2018; 10.

24. Li M, Su Y, Yu Y, et al. Dual roles of calpain in facilitating Coxsackievirus B3 replication and prompting inflammation in acute myocarditis. Int J Cardiol 2016; 221: 1123-31.

25. Yu Y, Shi H, Yu Y, et al. Inhibition of calpain alleviates Coxsackievirus B3-induced myocarditis through suppressing the canonical NLRP3 inflammasome/caspase-1-mediated and noncanonical caspase-11-mediated pyroptosis pathways. Am J Transl Res 2020; 12: 1954-64.

26. Britti E, Delaspre F, Tamarit J, Ros J. Calpain-inhibitors protect frataxin-deficient dorsal root ganglia neurons from loss of mitochondrial $\mathrm{Na}+/ \mathrm{Ca} 2+$ exchanger, NCLX, and apoptosis. Neurochem Res 2021; 46: 108-19.

27. Rivadeneyra L, Charó N, Kviatcovsky D, de la Barrera S, Gómez RM, Schattner M. Role of neutrophils in CVB3 infection and viral myocarditis. J Mol Cell Cardiol 2018; 125: 14961.

28. Wang C, Dong C, Xiong S. IL-33 enhances macrophage M2 polarization and protects mice from CVB3-induced viral myocarditis. J Mol Cell Cardiol 2017; 103: 22-30.

29. Gu X, Li Y, Chen K, et al. Exosomes derived from umbilical cord mesenchymal stem cells alleviate viral myocarditis through activating AMPK/mTOR-mediated autophagy flux pathway. J Cell Mol Med 2020; 24: 7515-30.

30. Xu H, Liu G, Gu H, Wang J, Li Y. Glutamine protects intestine against ischemia-reperfusion injury by alleviating endoplasmic reticulum stress induced apoptosis in rats. Acta Cir Bras 2020; 35: e202000104.

31. Jo HJ, Yang JW, Park JH, et al. Endoplasmic reticulum stress increases DUSP5 expression via PERK-CHOP pathway, leading to hepatocyte death. Int J Mol Sci 2019; 20.

32. Liu D, Zhang M, Yin H. Signaling pathways involved in endoplasmic reticulum stress-induced neuronal apoptosis. Int J Neurosci 2013; 123: 155-62.

33. Guan L, Che Z, Meng X, et al. MCU up-regulation contributes to myocardial ischemia-reperfusion injury through calpain/OPA1-mediated mitochondrial fusion/mitophagy inhibition. J Cell Mol Med 2019; 23: 7830-43.

34. Takahashi M, Tanonaka K, Yoshida H, et al. Possible involvement of calpain activation in pathogenesis of chronic heart failure after acute myocardial infarction. J Cardiovasc Pharmacol 2006; 47: 413-21.

35. Yu CG, Bondada V, Joshi A, et al. Calpastatin overexpression protects against excitotoxic hippocampal injury and traumatic spinal cord injury. J Neurotrauma 2020; 37: 2268-76.

36. Hu X, Wang H, Lu W, Dong Y, Cheng P. The study on intramyocardial calcium overload and apoptosis induced by Coxsackievirus B3. J Tongji Med Univ 2001; 21: 256-8, 262.

37. Su BC, Li CC, Horng JL, Chen JY. Calcium-dependent calpain activation-mediated mitochondrial dysfunction and oxidative stress are required for cytotoxicity of Epinecidin-1 in human synovial sarcoma SW982 cells. Int J Mol Sci 2020; 21.

38. Storr SJ, Carragher NO, Frame MC, Parr T, Martin SG. The calpain system and cancer. Nat Rev Cancer 2011; 11: 364-74.

39. Li Y, Guo Y, Tang J, Jiang J, Chen Z. New insights into the roles of CHOP-induced apoptosis in ER stress. Acta Biochim Biophys Sin (Shanghai) 2014; 46: 629-40. 
40. Cai Z, Shen L, Ma H, et al. Involvement of endoplasmic reticulum stress-mediated C/EBP homologous protein activation in Coxsackievirus B3-induced acute viral myocarditis. Circ Heart Fail 2015; 8: 809-18.

41. Dai Q, Zhang D, Yu H, et al. Berberine restricts Coxsackievirus B type 3 replication via inhibition of c-Jun $\mathrm{N}$-terminal kinase (JNK) and p38 MAPK activation in vitro. Med Sci Monit 2017; 23: $1448-55$.

42. Zhang Y, Ren S, Liu Y, Gao K, Liu Z, Zhang Z. Inhibition of starvation-triggered endoplasmic reticulum stress, autophagy, and apoptosis in ARPE-19 cells by taurine through modulating the expression of calpain-1 and calpain-2. Int J Mol Sci 2017; 18.

43. Zheng D, Wang G, Li S, Fan GC, Peng T. Calpain-1 induces en- doplasmic reticulum stress in promoting cardiomyocyte apoptosis following hypoxia/reoxygenation. Biochim Biophys Acta 2015; 1852: 882-92.

44. Yan M, Chen K, He L, Li S, Huang D, Li J. Uric acid induces cardiomyocyte apoptosis via activation of calpain-1 and endoplasmic reticulum stress. Cell Physiol Biochem 2018; 45: 212235.

45. Li S, Ma J, Li JB, et al. Over-expression of calpastatin attenuates myocardial injury following myocardial infarction by inhibiting endoplasmic reticulum stress. J Thorac Dis 2018; 10: 5283-97. 